\title{
Gestão de risco e as estratégias do plano de contingência para COVID-19
}

\author{
COVID-19 contingency plan strategies and risk management \\ Gestión de riesgos y estratégias del plan de contingencia para COVID-19
}

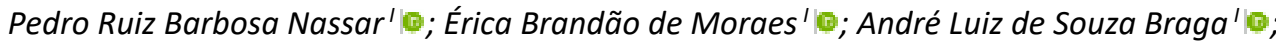 \\ Deise Ferreira de Souza' $\bullet$; Bárbara Pompeu Christóvam' ${ }^{\circ}$; Mercedes Neto "C
}

'Universidade Federal Fluminense, Niterói, RJ, Brasil; "Universidade do Estado do Rio de Janeiro, Rio de Janeiro, RJ, Brasil

\begin{abstract}
RESUMO
Objetivo: analisar o plano de contingência para infecção humana pelo Covid-19 e apresentar um modelo conceitual de gestão de risco para o Covid-19. Método: estudo de avaliação executiva, com análise seguindo os passos: Descrição da política; diagnóstico do problema; desenho da política; implementação; governança; resultados e impactos; que permitiu estabelecer o panorama geral acerca do Plano de Contingência Nacional para Infecção Humana pelo novo Coronavírus. Resultados: foram utilizados os passos metodológicos para apontar pontos positivos e fragilidades do plano de contingência e a construção de um modelo conceitual sobre a gestão de risco para o COVID-19. Conclusão: o cenário nacional enriquecido de condições socioambientais desfavoráveis, expõe o quão é vulnerável a nossa população e o sistema de saúde. Além disso, o estudo apontou para déficits de pessoal, materiais e preparação prévia para situações de risco como fatores a serem tratados dentro do processo de mitigação dos riscos.
\end{abstract}

Descritores: Infecções por Coronavírus; Gestão de riscos; Saúde Pública; Políticas Públicas de Saúde.

\begin{abstract}
Objective: to examine the contingency plan for human infection by Covid-19 and present a conceptual model of risk management for Covid-19. Method: in this executive evaluation study, the analysis followed the steps: policy description; problem diagnosis; policy design; implementation; governance; results and impacts; to establish an overall panorama of the National Human Infection Contingency Plan for the new Coronavirus. Results: the methodological steps were used to highlight the strengths and weaknesses of the contingency plan, and to construct a conceptual model of risk management for COVID-19. Conclusion: the scenario in Brazil, enhanced by unfavorable socio-environmental conditions, exposed how vulnerable its population and the health system are. The study also indicated that deficits in personnel, material and prior preparation for risk situations were factors to be addressed in the risk mitigation process.

Descriptors: Coronavirus Infections; Risk Management; Public Health; Public Health Policy.

\section{RESUMEN}

Objetivo: examinar el plan de contingencia para la infección humana por Covid-19 y presentar un modelo conceptual de gestión de riesgos para Covid-19. Método: en este estudio de evaluación ejecutiva, el análisis siguió los pasos: descripción de la política; diagnóstico de problemas; diseño de políticas; implementación; gobernancia; resultados e impactos; Establecer un panorama general del Plan Nacional de Contingencia de Infección Humana por el nuevo Coronavirus. Resultados: los pasos metodológicos se utilizaron para resaltar las fortalezas y debilidades del plan de contingencia y para construir un modelo conceptual de gestión de riesgos para COVID-19. Conclusión: el escenario en Brasil, potenciado por condiciones socioambientales desfavorables, expuso la vulnerabilidad de su población y el sistema de salud. El estudio también indicó que los déficits en personal, material y preparación previa para situaciones de riesgo fueron factores a ser abordados en el proceso de mitigación de riesgos. Descriptores: Infecciones por Coronavirus; Gestión de Riesgos; Salud Pública; Políticas Públicas de Salud.
\end{abstract}

\section{INTRODUÇÃO}

Uma das preocupações do século XXI relativo às emergências em saúde pública, relacionam-se aos eventos climatológicos e aparecimento de doenças ditas emergentes e reemergentes, que contribuem de forma expressiva com a morbimortalidade na sociedade atual. Tal fato é intensificado pelo cenário mundial de vulnerabilidade socioambiental que, de maneira direta, amplia o risco de impacto à saúde humana e os sistemas econômicos ${ }^{1,2}$.

Destaca-se que as doenças emergentes e reemergentes são aquelas consideradas doenças infecciosas cuja incidência em humanos permanece em aumento nos últimos anos, e que tem risco relevante de aumentar em futuro próximo. Diante dessas inquietações, estes tipos de doenças permanecem no rol de prioridades da Agenda Global de Saúde Pública, com alertas da comunidade científica, no desenvolvimento de pesquisas e reflexões para construção de conhecimentos ${ }^{3}$. 
Dentro do cenário de risco voltado para situações, que culminaram em grandes impactos na vida humana e econômicos, nos últimos cem anos testemunhou-se aproximadamente um elevado número, de 40 a 50 milhões de mortes, ocasionadas por pandemias e em sua maioria, por alterações genéticas como a do vírus Influenza (H1N1), Ebola e, em menor escala, pelo Coronavírus. Estima-se que, anualmente, 290 a 650 mil pessoas morrem por complicações oriundas das síndromes gripais ${ }^{4}$.

Embora ressoe como um cenário não planejado, de acordo com a Organização Mundial da Saúde (OMS), uma emergência em saúde pública caracteriza-se como uma situação que requer ações urgentes de medidas preventivas, de controle e contenção de riscos, danos e agravos à saúde humana. Tais situações podem ser oriundas de epidemias, desastres naturais e falta de assistência a um determinado grupo ou população².

Em dezembro de 2019, em Wuhan, na China, ocorreu o surgimento do novo Coronavírus (COVID-19) causando uma pandemia de pneumonia viral incomum, que levou a comunidade mundial a declarar estado de calamidade/emergência em saúde pública ${ }^{5-6}$. Deflagrado pelo cenário mundial, em janeiro de 2020, foi ativado o Centro de Operações de Emergências em Saúde Pública para o novo Coronavírus (COE-nCoV). No mesmo mês, o primeiro caso alóctone suspeito foi notificado, elevando o nível de atenção mundial ${ }^{7,8}$.

Dentre os planos necessários ao enfrentamento da pandemia, o Ministério da Saúde elaborou o Plano de Contingência Nacional para Infecção Humana pelo novo Coronavírus com o objetivo de conter a infecção humana e mitigar o aparecimento de casos graves e óbitos ocasionados pelo novo Coronavírus. Este plano é composto por três níveis de resposta: Alerta, Perigo Iminente e Emergência em Saúde Pública. Cada nível é baseado na avaliação do risco do COVID-19 afetar o Brasil e seu impacto à saúde pública9 ${ }^{9}$.

No cenário atual de pandemia, ainda há muitas dificuldades de controle e redução da morbimortalidade pelas diferentes manifestações do Coronavírus, pela dificuldade de acesso ao sistema de saúde, pela falta de medicamentos eficazes e por ausência de vacinas, ainda em fase de testes, tornando-se necessário a permanência de ações voltadas à Gestão de Risco. Portanto, tais atuações inserem: planejar ações para redução de novos casos, através do isolamento social e medidas de educação em saúde voltadas à população; programar políticas públicas que assegurem a vida e a segurança da população; propiciar que gestores e profissionais desenvolvam ações de gestão do cuidado em saúde, alinhadas aos protocolos internacionais, a fim de garantir proteção dos trabalhadores de saúde e implementação de ações seguras e de qualidade aos pacientes. A adoção dessas estratégias visa também o aumento da capacidade assistencial do sistema de saúde que dê suporte ao impacto decorrente da pandemia ${ }^{10}$.

Entretanto, considerando-se que o adoecimento e a mortalidade da população envolvem não só os idosos e doentes crônicos, ditos grupo de maior risco, mas também população de adultos jovens, que houve considerável comprometimento da saúde e perda de profissionais de saúde, que há um processo crônico de gestão pública ineficaz, envolvendo déficit de capital humano, materiais e mau gerenciamento de leitos públicos, esse estudo centra-se nas questões: A política traçada para enfrentamento do coronavírus apresenta-se eficaz diante do número de novos casos e de mortes crescentes em vários estados ou flutuam entre a estabilidade e aumento? Atende a todo território nacional que tem diferentes contextos? As falhas estão no seu planejamento, acompanhamento ou na execução? No tempo que demorou para ser executada, na incredulidade do risco do problema de saúde pública?

Neste contexto, o presente estudo tem como objetivo analisar o plano de contingência para infecção humana pelo COVID-19 e apresentar um modelo conceitual de gestão de risco para o COVID-19

\section{REFERENCIAL TEÓRICO}

As políticas públicas devem ser entendidas como um conjunto de medidas, representadas através de programas, planos e metas, criadas pelos governos, voltadas à garantia de acesso pela população aos direitos estabelecidos por Lei, tais como a assistência ou prestação de serviços. Essas medidas integram parte importante da administração pública, já que representam as ações dos governos criadas para melhor atender as necessidades dos cidadãos ${ }^{11-13}$.

O risco é um fenômeno coletivo, que atinge uma população. Na saúde pública, a o conceito de risco é aplicado na identificação de grupos populacionais que diferem entre si em relação à probabilidade de desenvolver eventos relativos à saúde. Este conceito é utilizado no planejamento e gestão, a partir da identificação dos grupos de maior exposição a fatores de risco à saúde ${ }^{14}$.

Na tentativa de preencher lacunas acerca do tema, foi adotado nesse estudo os aspectos de desenvolvimento e os vieses e desafios no contexto nacional. Essa abordagem avalia o cenário atual e correlaciona com estudos já disponíveis que tratam da temática de gestão de riscos em situações extremas. 


\section{MÉTODO}

A abordagem do estudo se deu através do método de avaliação executiva, que permite estabelecer o panorama geral acerca de determinada política pública, corroborando para a identificação de fragilidades e pontos relevantes que necessitem de aprimoramento a nível de gestão e/ou execução ${ }^{15}$.

Esse tipo de avaliação é composto pelo sequenciamento de ações metodológicas, sendo essas ações denominadas de "passos". Neste sentido, a pesquisa teve como trajetória os seguintes passos: descrição da política; diagnóstico do problema; desenho da política; implementação; governança; resultados; impactos e proposições ${ }^{15}$.

Na presente análise, construiu-se o corpus a partir da nossa relação com o tema relevante, tendo como princípio o Plano de Contingência Nacional para Infecção Humana pelo novo Coronavírus. A coleta e organização dos dados se deu através das portarias no 188, de 3 de fevereiro de 2020 e no 356, de 11 de março de 2020, além do Plano de Contingência Nacional para Infecção Humana pelo novo Coronavírus, publicado em janeiro de $2020^{16}$

A partir da descrição da política como instrumento a ser avaliado, partiu-se para a correlação do cenário nacional e desafios socioambientais, econômicos e de gestão. Essa abordagem facilita o processo de análise por permitir a interseção entre o cenário atual de pandemia, os desafios conhecidos e observados e a continuidade nos passos metodológicos propostos. A abordagem possibilitou a correlação com a gestão de riscos, colaborando com a proposição final com base na avaliação utilizada.

Neste sentido os passos se delinearam da seguinte forma: Descrição da política - O plano de contingência para o COVID-19; diagnóstico do problema - cenário observado; desenho da política - as ações de gestão; implementação análise dos desdobramentos após a implementação das políticas; governança - impacto social pela capacidade de governar; resultados e impactos - consequências da má gestão e governança frágil; proposições - articular gestão de risco e a pandemia pelo COVID-19.

\section{RESULTADOS}

\section{Descrição da política - 0 plano de contingência para o COVID-19}

Em 30 de janeiro de 2020, o Diretor Geral da OMS declarou o surto de 2019 como uma emergência saúde pública de preocupação internacional. Foram emitidas recomendações temporárias à República Popular da China e a outros países. Para tanto, elaborou-se um plano estratégico de preparação e resposta no controle e enfrentamento do COVID19 para os países já afetados, mas também, para mitigar o impacto do surto em todos os países.

O plano de contingência tem como propósito, organizar hierarquicamente os níveis de tomada de decisão e responsabilidade institucional, além de nortear todas as instancias sobre normas e condutas. Os objetivos estratégicos para dar resposta ao plano foram: Limitar a transmissão humano a humano, incluindo a redução infecções secundária entre contatos próximos e assistência médica aos trabalhadores, prevenindo eventos de amplificação de transmissão, e impedindo uma maior disseminação internacional da China; Identificar, isolar e cuidar dos pacientes desde o início dos sintomas, incluindo fornecer atendimento otimizado para pacientes infectados; Abordar incógnitas cruciais sobre a gravidade clínica extensão da transmissão e infecção, opções de tratamento, e acelerar o desenvolvimento de diagnósticos, terapêutica e vacinas; Comunicar informações críticas sobre riscos e eventos a todas as comunidades e combater a desinformação e Minimizar o impacto social e econômico através de parcerias multissetoriais ${ }^{9}$.

\section{Diagnóstico do Problema - cenário observado}

A segunda fase de diagnóstico, é uma correlação com ex post de concepção da política ainda é válida diante de novos contextos, ou seja, se o problema permanece e se as suas causas são ainda as anteriormente levantadas. $\mathrm{O}$ que de fato ocorreu e ainda ocorre no cenário nacional da pandemia do novo coronavírus. Determinando a necessidade do estado de emergência em saúde pública. Entretanto, 08 meses após o surgimento da pandemia, os dados de infectados e óbitos, ultrapassam as expectativas iniciais, bem como o tempo de duração da pandemia.

Tais objetivos, para serem alcançados necessitariam estabelecer rapidamente uma coordenação internacional para fornecer suporte estratégico, técnico e operacional através de mecanismos e parcerias existentes; ampliar a preparação e a resposta operacionais dos países, incluindo o reforço da prontidão para identificar, diagnosticar e tratar casos; prevenção e controle de infecção em ambientes de saúde; implementação de medidas de saúde para viajantes; e conscientização da população, ou seja, comunicação de risco para ampliar o envolvimento da comunidade. Acelerar pesquisas e inovações prioritárias para apoiar um processo global claro e transparente, desenvolvimento e disponibilidade equitativa terapêutica, vacinas e diagnósticos ${ }^{17}$. 


\section{Desenho da Política - as ações de gestão}

A análise do desenho da política considera, em linhas gerais, três elementos: modelo lógico, incentivos existentes e forma de acesso à política. O modelo partiu nas recomendações da OMS, que baseou suas estratégias de resposta em várias premissas, sua transmissibilidade e o espectro clínico da doença, necessitariam de atualizações regulares a fim de preencher lacunas do conhecimento da doença e seu potencial de disseminação. Os incentivos econômicos e sociais foram estabelecidos, porém, inúmeros problemas foram noticiados como: atraso nas obras de hospitais de campanha, falta de insumos, programas sociais que não abrangeram toda a população vulnerável nesse cenário e a omissão do MS em disponibilizar os dados, levando ao pensamento epidemiológico de subnotificação dos casos, o que gera incerteza e desconfiança no cenário político, econômico e social ${ }^{18}$.

Com a propagação do COVID-19 no mundo, de forma escalonada, no Brasil foi possível observar os primeiros comportamentos do vírus em outros países da Ásia e Europa, antes de sua entrada no país. Esta oportunidade permitiu que o Ministério da Saúde pudesse elaborar estratégias preventivas para esse enfrentamento que visaram, apesar de algumas incertezas, reduzir ao máximo o número de infectados sintomáticos ou não, evitando um colapso na rede hospitalar pública.

\section{Implementação - análise dos desdobramentos após a implementação das políticas}

Mediante ao exposto, o quarto passo da avaliação referente a implementação, foi possível identificar estratégias positivas quanto ao isolamento social recomendado, a construção de ambientes temporários em caráter de emergência (gabinetes de crise, grupos de trabalho, hospitais de campanha e aquisição de materiais e insumos). No decorrer dos meses, foi possível identificar falha nos processos e na comunicação, sendo utilizado a verticalização hierárquica a nível político para a tomada de decisão e o descumprimento de protocolos e recomendações.

Devido a embates políticos, a vulnerabilidade social que faz parte do cotidiano das comunidades carentes, tribos indígenas, municípios no interior do país e com graves problemas sócio econômicos, o processo de isolamento foi fragilizado, potencializando os desafios que emergiram com a pandemia, o que debilita o quinto passo que é a análise da governança nesse cenário.

\section{Governança - impacto social pela capacidade de governar}

A estratégia do governo, que visa auxiliar a população menos favorecida, com a manutenção do isolamento social, teve cenários desfavoráveis como: a não contemplação de parte da população que necessita, fraudes e desgastes pela exposição em grandes filas, como noticiado pelos veículos de imprensa, além de parte do isolamento fragilizado pelo discurso de governantes $\mathrm{O}$ que implica diretamente na economia, que não suportará por muito tempo e se desdobrará em uma crise financeira sem precedentes.

\section{Resultados e impactos - consequências da má gestão e governança frágil}

Ao se analisar os resultados e impactos, alguns pontos devem ser levados em conta, como por exemplo: os impactos da não testagem em massa o que causa a subnotificação e falta de clareza no perfil da doença e como ela se comporta no cenário nacional que é multivariado. Haja vista os números noticiados de casos notificados, que até meados do mês de agosto ultrapassaram os 3 milhões de infectados e mais de 100 mil óbitos, o que de certa forma não demonstra a realidade do cenário brasileiro ${ }^{19}$.

No que tange as questões sobre o gasto orçamentário, foi observado uma janela de fragilidades inerentes ao processo de aquisição de materiais e insumos, contratos fraudulentos e a não operacionalização de alguns hospitais de campanha. O mal-uso de verba pública em aquisição e contratos superfaturados, bem como o desvio de verba impacta de maneira negativa tanto no âmbito político, quanto na qualidade da assistência prestada a saúde da população.

\section{Proposições - articular gestão de risco e a pandemia pelo COVID-19}

A Análise dos dados se deu de maneira descritiva e analítica, baseado na avaliação executiva. Foram utilizados estudos sobre a gerência de risco em saúde possibilitando a interpretação do cenário atual e possíveis inferências no âmbito da gestão em saúde. A análise possibilitou como técnica de pesquisa, a construção de inferências válidas e reproduzíveis de acordo com sua especificidade ${ }^{20}$.

Na presente análise, construiu-se uma correlação a partir da relação de políticas públicas de saúde e gestão de risco em saúde, como instrumentos para o processo de gestão e tomada de decisão. Temas relevantes dentro do contexto atual de saúde e de pandemia.

O curso de uma pandemia como a atual é permeado de incertezas que devem ser reavaliadas periodicamente, à medida que novas descobertas na ciência são divulgadas. Recentemente foram pontuadas 4 incertezas sobre o COVID19, que impactam na gestão dos riscos associados. A primeira incerteza e refere à taxa de letalidade da doença. Para que seja calculada com precisão, são necessárias informações sobre o número de pessoas infectadas. Em grande parte 
dos países, testes em larga escala não foram realizados e, além disso, existem óbitos registrados sem causalidade definida para o COVID-19. Dessa forma, pode haver valores subestimados ou superestimados dessa variável ${ }^{21}$.

A segunda incerteza é sobre o período de transmissão da doença, antes dos primeiros sintomas. Considerando um tempo de incubação médio de 5 a 6 dias, os gestores enfrentam o desafio de como conter a transmissão, no período em que ela ainda é assintomática. A terceira incerteza é o reflexo da segunda e envolve o número de casos assintomáticos da doença. Partindo do pressuposto que $80 \%$ dos casos não desenvolvem sintomas, medidas de isolamento social e lockdown podem promover redução da disseminação, contudo, com grande impacto econômico e social. Por último, a quarta incerteza que permeia a tomada de decisão, é referente a duração da infecção, e risco de transmissão pós quarentena ${ }^{21}$.

Como medidas para gerenciar os riscos, a combinação de atividades de contenção e mitigação tem sido utilizadas, com objetivo de evitar o pico da epidemia e sobrecarga do sistema de saúde por um período prolongado, proteger populações vulneráveis, bem como reduzir a morbidade e mortalidade. Ações gerenciais para atingir esses objetivos variaram e devem ser ponderadas com base no risco e avaliação do contexto nacional. O Brasil vivencia atualmente a pandemia do COVID-19 e de maneira imprudente propagou informações e induziu a comportamentos que vão contra as recomendações da OMS, que estão fundamentadas em resultados obtidos em outros países e pesquisas científicas recentes ${ }^{22}$.

Avaliar o contexto interno é uma etapa fundamental no sistema de gestão de riscos, para determinar ações direcionadas. É importante destacar peculiaridades nas características populacionais do Brasil, pois é um país cuja população consiste principalmente de adultos jovens. Entretanto tem-se elevada prevalência de comorbidades relacionadas ao agravamento dos casos do COVID-19, como hipertensão, diabetes, obesidade, tuberculose, dentre outras. Nesse sentido, o enfoque a partir da atual pandemia no Brasil, precisa estar direcionado às populações mais vulneráveis, no que tange a preparação para novos cenários como este 22 .

A maior oferta de leitos e a gestão da superlotação dos serviços de saúde é outra medida que deve ser implementada. Isso implica em maior disponibilidade de leitos de UTI e aquisição de equipamentos de forma emergencial. O que em alguns cenários se mostrou fragilizado pela falta de planejamento prévio, agilidade na tomada de decisão e gestão pública fraudulenta e ineficaz ${ }^{21}$.

A promoção de medidas de saúde pública, com reforço na educação de profissionais de saúde, na prevenção e controle de infecção por COVID-19, foi fundamental. Além disso, grande parte da população recebeu orientações sobre quais medidas de prevenção tomar e cada situação de exposição, além de como prover o isolamento domiciliar dos casos leves. O que ainda é um desafio, haja vista as diferentes realidades habitacionais dentro do cenário nacional ${ }^{21}$.

\section{Proposta de Modelo de Gestão de Risco em emergências em saúde pública: COVID-19}

Neste sentido, devido as fragilidades e desafios no que tange a gestão do cuidado em saúde em situações de emergência em saúde pública, algumas peculiaridades estão sendo observadas, como por exemplo: a não realização de teste massivamente, o que de certo fragiliza os dados epidemiológicos para tomada de decisão; o déficit de leitos hospitalares de terapia intensiva, acarretando em aumento da espera por internação, colapso do sistema de saúde e aumento do número de óbitos; a escassez de material, ocasionado pela demanda global; o déficit de capital humano qualificado, um problema já existente na rede pública de saúde, que corroborado pelos milhares de afastamentos de profissionais infectados impactam mais na qualidade da assistência.

Para tanto, como proposta de fomentar boas práticas de gestão em situações de calamidade, traz-se como exemplo o esquema conceitual adaptado do estudo de Nassar (2017) e Nassar e Porto (2019), como possibilidade de nortear os subprocessos da gestão nesse cenário. Esses subprocessos são a análise do ambiente, seja ele interno: Intra hospitalar (capacidade de leitos e possíveis adequações), Atenção Primária à Saúde - APS (estruturas e fluxos) e externo (condições socioambientais e hospitais de campanha); Gerenciamento do cuidado direto (cuidado aos pacientes infectados e suspeitos, comunidades pela APS, manejo de cadáveres) e indireto (insumos, serviço de vigilância epidemiológica em saúde, tecnologia de equipamentos e análise de exames específicos e educação permanente para capacitação de profissionais); Planejamento e organização (nível central e regional com gabinetes de crise e centro de operações, grupos de trabalho institucionais e elaboração de processos gerenciais diretos e indiretos).

A elaboração de instrumentos administrativos: como manuais e protocolos de manejo clínico, segurança do paciente e profissional, boletins epidemiológicos atualizados) são necessários; os aspectos culturais envolvem (comportamento coletivo e vulnerabilidade que impactam diretamente na diminuição da curva de infectados e exposição ao agente patológico, cultura organizacional das instituições de saúde, que ditam diretamente quanto determinada instituição consegue se adequar ao novo cenário) e por fim o corpo (que tanto na concepção fisiopatológica determina os casos a serem acompanhados pela rede básica de saúde, no domicilio ou no ambiente intra-hospitalar, quanto nos aspectos psicológicos no enfrentamento de situações de medo, pânico e angustia, ocasionados pelas incertezas e isolamento social). 
Em detrimento do exposto, apresenta-se o esquema conceitual (Figura 1) de gestão de risco, a ser aplicado em situações extremas como a vivida na pandemia do novo Coronavírus.

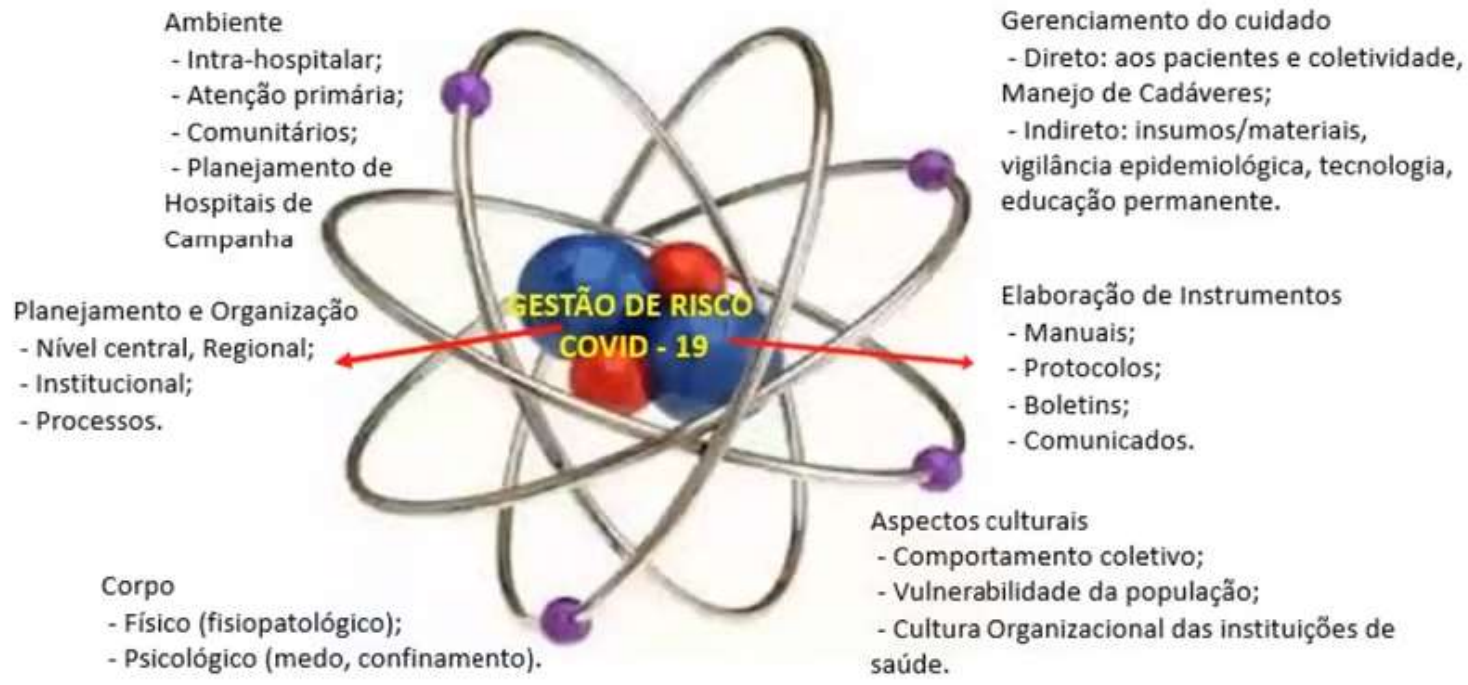

Fonte: Adaptado de Nassar (2017) e Nassar e Porto (2019).

FIGURA 1: Esquema conceitual para Gestão de Risco COVID-19. Niterói, RJ, Brasil, 2020.

\section{CONCLUSÃO}

O estudo permitiu a análise do Plano de Contingência Nacional para Infecção Humana pelo novo Coronavírus, que apontou para algumas fragilidades no âmbito da gestão de risco nesse cenário. O cenário nacional enriquecido de condições socioambientais desfavoráveis, expõe o quão é vulnerável a nossa população e o sistema de saúde.

Os processos gerencias desde os níveis centrais, até o âmbito das instituições de saúde, apontam para déficits de pessoal, materiais e preparação prévia para situações de risco. Esses desafios nos levam a reflexão da atual necessidade de preparação para eventos de calamidade pública e emergência em saúde pública. Sendo de extrema relevância modelos de gestão inseridos nesse contexto.

Para tanto, se torna singular o comprometimento tanto de gestores, colaboradores e quanto da população, para a efetivação das políticas públicas e em estratégias norteadoras como o plano de contingência, para que o país consiga combater a pandemia, com níveis aceitáveis de morbimortalidade e não ocorra a sobrecarga dos serviços de saúde. Tornando viável a eficiência das ações em prol da saúde da população brasileira.

\section{REFERÊNCIAS}

1. Tabata KI, Ito K, Pirondi ACS, Mori AS. Benefits of breastfeeding in reducing the number of hospitalizations in children under two years old. Braz J of Develop [Internet], 2019 [cited 2020 Jan 20]; 5(11):27995-8010. DOI: https://doi.org/10.34117/bjdv5n11-388.

2. Victora CG, Bahl R, Barros AJ, França GV, Horton S, Krasevec J, et al. Breastfeeding in the 21st century: epidemiology, mechanisms, and lifelong effect. Lancet [Internet], 2016 [cited 2020 Jan 20]; 387(10017):475-90. DOI: https://doi.org/10.1016/S0140-6736(15)01024-7.

3. Rodrigues SM, Lima OF. Aleitamento materno é mais que um direito: um benefício para toda a família. ReBIS [Internet], 2019 [cited 2020 Feb 15]; 1(1): 1-8. Available from: http://revista.rebis.com.br/index.php/rebis/article/view/121.

4. Lima APC, Nascimento DS, Martins MMF. The practice of breastfeeding and the factors that take to early weaning: an integrating review. Rev. J. Health Biol. Sci. [Internet], 2018 [cited 2020 Feb 15]; 6(2):189-96. DOI: http://dx.doi.org/10.12662/2317-3076jhbs.v6i2.1633.p189-196.2018.

5. Boccolini PMM, Monteiro FB, Venâncio SI, Giugliani ERJ. Breastfeeding indicators trends in Brazil for three decades. Rev. Saúde Pública [Internet], 2017 [cited 2020 Feb 15]; 51:108. DOI: https://doi.org/10.11606/S1518-8787.2017051000029.

6. Moore ER, Bergman N, Anderson GC, Medley N. Early skin-to-skin contact for mothers and their healthy newborn infants. Cochrane Database Syst. Rev. [Internet], 2012 [cited 2020 Feb 15]; 5:CD003519. DOI: https://doi.org/10.1002/14651858.CD003519.pub3. 
7. Kologeski TK, Strapasson MR, Schneider V, Renosto JM. Skin to skin contact of the newborn with its mother in the perspective of the multiprofessional team. Rev. Enferm. UFPE Online [Internet], 2016 [cited 2020 Feb 15]; 11(1):94-101. Available from: https://periodicos.ufpe.br/revistas/revistaenfermagem/article/download/11882/14340.

8. Moraes BA, Gonçalves AC, Strada JKR, Gouveia HG. Factors associated with the interruption of exclusive breastfeeding in infants up to 30 days old. Rev. Gaúcha Enferm. [Internet], 2016 [cited 2020 Feb 15]; 37(spe):e2016-0044. DOI: https://doi.org/10.1590/1983-1447.2016.esp.2016-0044.

9. Silva OLO, Rea MF, Sarti FM, Silva MO. Association between infant formula and pacifier supply in maternity and breastfeeding in the first six months of life. DEMETRA [Internet], 2019 [cited 2020 Feb 15]; 1:e43555. DOI: https://doi.org/10.12957/DEMETRA.2019.43555.

10. Pinheiro JMF, Menêzes TB, Brito KMF, Melo ANL, Queiroz DJM, Sureira TM. Prevalence and factors associated with the prescription/request for infant formula. Rev. Nutr. [Internet], 2016 [cited 2020 Feb 15]; 29 (3):367-75. DOI: https://doi.org/10.1590/1678-98652016000300007.

11. Lamounier JA, Chaves RG, Rego MAS, Bouzada MCF. Baby friendly hospital initiative: 25 years of experience in Brazil. Rev. paul. pediatr. [Internet], 2019 [cited 2020 Feb 15]; 37(4):486-93. DOI: https://doi.org/10.1590/1984-0462/;2019;37;4;00004.

12. Berde AS, Yalcin SS. Determinants of early initiation of breastfeeding in Nigeria: a population-based study using the 2013 demograhic and health survey data. BMC Pregnancy Childbirth [Internet], 2016 [cited 2020 Feb 15]; 16(1):32. DOI: https://doi.org/10.1186/s12884-016-0818-y.

13. Saco MC, Coca KP, Marcacine KO, Abuchaim ÉSV, Abrão ACFV. Skin-to-skin contact followed by breastfeeding in the first hour of life: associated factors and influences on exclusive breastfeeding. Texto contexto - enferm. [Internet], 2019 [cited 2020 Feb 15]; 28:e20180260. DOI: https://doi.org/10.1590/1980-265x-tce-2018-0260.

14. Silva DD, Schmitt IM, Costa R, Zampieri MFM, Bohn IE, Lima MM. Promotion of breastfeeding in prenatal care: the discourse of pregnant women and health professionals. REME [Internet], 2018 [cited 2020 Feb 15]; 22:e-1103. DOI: https://doi.org/10.5935/1415-2762.20180031.

15. Santos JB, Souza EN, Rocha CS, Trindade FS, Oliveira KA. Aspectos epidemiológicos do parto cesáreo em Sergipe. Rev. Saúde ReAGES [Internet], 2019 [cited 2020 Feb 15]; 1(4):47-51. Available from: http://npu.faculdadeages.com.br/index.php/revistadesaude/article/view/168.

16. Silva ACL, Félix HCR, Ferreira MBG, Wysocki AD, Contim D, Ruiz MT. Preference for type of childbirth, factors associated with expectation and satisfaction with childbirth. Rev. Eletr. Enf. [Internet], 2017 [cited 2020 Feb 15]: 19-34. DOI: https://doi.org/10.5216/ree.v19.44139.

17. Arruda GT, Barreto SC, Morin VL, Petter GN, Braz MM, Pivetta HMF. Is there a relation between mode of delivery and breastfeeding in the first hour of life? Rev. Bras. Promoç. Saúde [Internet], 2018 [cited 2020 Feb 15]; 31(2):1-7. DOI: https://doi.org/10.5020/18061230.2018.7321.

18. Alzaheb RA. A review of the factors associated with the timely initiation of breastfeeding and exclusive breastfeeding in the Middle East. Clin. Med. Insights Pediatr. [Internet], 2017 [cited 2020 Feb 15]; 11. DOI: https://doi.org/10.1177/1179556517748912.

19. Silva CM, Pellegrinelli ALR, Pereira SCL, Passos IR, Santos LC. Educational practices in accordance with the "Ten steps to successful breastfeeding" in a Human Milk Bank. Ciênc. Saúde Colet. [Internet], 2017 [cited 2020 Feb 15]; 22(5):1661-71. DOI: https://doi.org/10.1590/1413-81232017225.14442015.

20. Silva CM, Pereira SCL, Passos IR, Santos, LC. Factors associated with skin to skin contact between mother/son and breastfeeding in the delivery room. Rev. Nutr. [Internet], 2016 [cited 2020 Feb 15]; 29(4):457-71. DOI: https://doi.org/10.1590/167898652016000400002.

21. Lau Y, Tha PH, Ho-Lim SST, Wong LY, Lim PI, Citra Nurfarah BZM, et al. An analysis of the effects of intrapartum factors, neonatal characteristics, and skin-to-skin contact on early breastfeeding initiation. Matern. Child. Nutr. [Internet], 2017 [cited 2020 Feb 15]; 14(1): e12492. DOI: https://doi.org/10.1111/mcn.12492.

22. Silva JLP, Linhares FMP, Barros, AA, Souza, AG, Alves DS, Andrade PON. Factors associated with breastfeeding in the first hour of life in a baby-friendly hospital. Texto Contexto Enferm. [Internet], 2018 [cited 2020 Feb 15]; 27(4):e4190017. DOI: https://doi.org/10.1590/0104-07072018004190017.

23. Karim F, Billah S.M, Chowdhury MAK, Zaka N, Manu A, Arifeen SE, et al. Initiation of breastfeeding within one hour of birth and its determinants among normal vaginal deliveries at primary and secondary health facilities in Bangladesh: a case-observation study. PLoS ONE [Internet], 2018 [cited 2020 Feb 15]; 13(8):e0202508. DOI: https://doi.org/10.1371/journal.pone.0202508.

24. Linares AM, Wambach K, Rayens MK, Wiggins A, Coleman E, Dignan MB. Modeling the influence of early skin-to-skin contact on exclusive breastfeeding in a sample of hispanic immigrant women. J. Immigr. Minor Health [Internet], 2017 [cited 2020 Mar 15]; 19(5):1027-34. DOI: https://doi.org/10.1007/s10903-016-0380-8.

25. Kim B. Factors that influence early breastfeeding of singletons and twins in Korea: a retrospective study. Int. Breastfeed J. [Internet], 2016 [cited 2020 Mar 15]; 12(4). DOI: https://doi.org/10.1186/s13006-016-0094-5.

26. Smith ER, Hurt L, Chowdhury R, Sinha B, Fawzi W, Edmond KM, et al. Delayed breastfeeding initiation and infant survival: A systematic review and meta-analysis. PLoS ONE [Internet], 2017 [cited 2020 Mar 15]; 12(7):e0180722. DOI: https://doi.org/10.1371/journal.pone.0180722.

27. Calegari FL, Barbieratto BJ, Fujinaga $\mathrm{Cl}$, Fonseca $\mathrm{LMM}$, Oliveira $\mathrm{CR}$, Leite AM. Full-term newborns' readiness during the first breastfeeding in rooming-in. Rev. Rene [Internet], 2016 [cited 2020 Mar 15]; 17(4):444-50. Available from: http://periodicos.ufc.br/rene/article/view/4927. 